\title{
Modeling, metrics, and optimal design for solar energy-powered base station system
}

\author{
Heng Wang ${ }^{1,2}$, Hongjia $\mathrm{Li}^{2}$, Chaowei Tang ${ }^{1 *}$, Lingbao Ye ${ }^{2}$, Xin Chen ${ }^{2}$, Hui Tang ${ }^{1,2}$ and Song $\mathrm{Ci}^{2,3}$
}

\begin{abstract}
Using renewable energy system in powering cellular base stations (BSs) has been widely accepted as a promising avenue to reduce and optimize energy consumption and corresponding carbon footprints and operational expenditures for $4 \mathrm{G}$ and beyond cellular communications. However, how to design a reliable and economical renewable energy powering (REPing), while guaranteeing communication reliability, renewable energy utilization, and system durability, is still a great challenge. Motivated by this challenge, we firstly model the dynamic energy flow behavior of solar energy-powered BS by using stochastic queue model, jointly considering instability of solar energy generation, non-linear effects of energy storage, and time varies of traffic load. On the basis of the model, three key performance metrics, including service outage probability (SoP), solar energy utilization efficiency (SEuE), and mean depth of discharge (MDoD), are defined, and close-form expressions of them are derived. Finally, under the guidelines of defined metrics, the sizing optimization problem is formulated, and then we propose the capital expenditure (CAPEX) minimization algorithm to resolve it with considerations of communication reliability, efficiency, and durability. Numerical results conducted to demonstrate the effectiveness of our proposed metrics vividly showed the close relationship between design metrics and system parameters. Simulation results also showed that our proposed algorithm can reduce at least $12.1 \%$ CAPEX compared with the classic algorithms and guarantee SoP below $0.82 \%$, SEuE above $97 \%$, and MDoD ranging from $7.2 \%$ to $9.1 \%$, which means that the optimal design was achieved in terms of system reliability, efficiency, durability, and investment. The proposed modeling, design metrics, and sizing method provide a theoretical basis for actual designs of REPing BS system, which also can be further applied to the scenario of other forms of renewable energy powered system.
\end{abstract}

Keywords: Renewable energy; Green communication; Optimal design; Queuing theory

\section{Introduction}

With the rapidly growing demand for wireless data traffic in cellular networks, several orders of magnitude base stations (BSs) have been deployed, and thus relevant energy consumption has increased exponentially. The consumed energy is mainly generated by non-renewable fossil resources, such as coal and natural gas. Thus, the escalation of energy consumption has brought about the substantial increase of carbon dioxide emission which contributes to climate change and global warming. In addition to the environmental aspects, the ever-increasing fossil fuel price also attracts the network operators' attention to the operational expenditure (OPEX). According

\footnotetext{
*Correspondence: cwtang@cqu.edu.cn

${ }^{1}$ College of Communication Engineering, Chongqing University, 400044 Chongqing, China

Full list of author information is available at the end of the article
}

to statistics, energy consumption for each BS rises to 25 MWh per annum and energy expense per BS increases to $\$ 3,200$ per annum with a carbon footprint of 11 tons of $\mathrm{CO}_{2}[1]$.

Renewable resources, such as solar and wind, are envisaged to be green energy sources to power cellular BSs due to their pollution-free and renewable nature [2]. Therefore, BSs powered by renewable energy are drawing more and more attention from the academia and industry. For example, the 3rd Generation Partnership Project (3GPP) technique report [3] and technique specification [4] explicitly encourage the use of renewable energy resources in mobile networks. Companies, such as Ericsson and Alcatel-Lucent, have launched a plan to exploit renewable energy resources for off-grid BSs in rural or remote areas [5]. Some a priori arts [6,7] focus

\section{Springer}


on making renewable energy permeation into heterogeneous networks (HetNet) in cities, since small cells in HetNet (e.g., micro-, pico-, and femto-cells) are more feasible to be powered by renewable energy in terms of cost and actual engineering. Furthermore, renewableenergy-powered BS system also has raised concerns in particular scenarios, such as private wireless communication networks for power and petroleum industry, emergency wireless communication networks for disaster relief, etc.

The renewable-energy-powered BS system can be abstracted into the energy harvesting part, the energy storage part, and the energy consumption part, in which energy flow behavior is highly dynamic. In the energy harvesting part, electric energy, which is converted from renewable energy resources, is unstable. Taking solar energy as an example, the output energy of photovoltaic (PV) panels is highly dynamic since the ambient temperature and solar irradiance vary from time to time [8]. In the energy storage part, battery bank is utilized to store the generated electric energy and to supply energy to the BS. The charging and discharging processes are influenced by non-linear effects of the battery bank [9] (e.g., the current effect, recovery effect, and internal resistance effect). Meanwhile, economic factor restricts the size and capacity of the energy harvesting part and the energy storage part. Furthermore, the energy consumption part, i.e., the energy consumption of the BS, is related to plenty of random factors (e.g., users' distribution and service time, cell size, and quality-of-service (QoS) requirement) and is quite diverse. Therefore, one of the most challenging topics is how to design a reliable and efficient renewable-energy-powered cellular system, which is also the primary condition for practical deployments.

Nowadays, the main efforts of a priori arts have been put on energy-efficient transmission, networking and network deployment, etc. for cellular networks powered by renewable energy. For example, the adaptive resource management framework was proposed in [10] for relay path selection and admission control in wireless mesh networks with renewable energy supplies. The intelligent cell breathing was obtained in [11], which balanced the energy consumptions among BSs which powered by renewable energy according to their separate energy storages. In [12], the throughput maximization problem was investigated for the orthogonal relay channel with energyharvesting source and relay nodes. In [13], to improve network throughput, the link scheduling was studied for rechargeable wireless sensor networks. However, there are no a priori existing arts to well investigate the design issue of renewable energy powered BS system. Fortunately, the renewable-energy-powered BS system can be viewed as a special case of renewable-energy-powered system, and various design methods of the renewableenergy-powered system have been studied in literatures. The intuitive method was provided in [14], which calculated the size of the photovoltaic system using simple mathematical equations without considering the random nature of solar energy, but it may cause an under or oversizing of the designed system. In [15], a design metric named as loss of power supply probability (LPSP) was proposed to quantify the energy reliability of the system. It is defined as the long-term average fraction of the energy demand that is not supplied by the standalone photovoltaic system. Based on the LPSP, an iterative optimization method [16] was provided for a hybrid energy-powered system. Similarly, a stochastic method was given in [17], which identified the involved sizing parameters for the stand-alone photovoltaic system. For any given desired LPSP, the optimal combination of the battery bank and photovoltaic module can be achieved. In [18], an analytical method was firstly used to obtain a data set of optimum sizes of the photovoltaic system at different LPSP levels, and then an artificial neural network was employed to train this data set and to predict the optimum size of the system. However, there are no existing works analyzing the energy flow behavior and energy dynamics of the renewable energy powered system, not to mention that of the renewable-energy-powered BS system. Furthermore, most existing methods merely focus on the relationship between system reliability and investment, neglecting renewable energy utilization and system durability.

The following are some fundament problems that have still not been well studied:

1) How to model and analyze the energy flow behavior and energy dynamics of the renewable-energy-powered cellular BS system;

2) How to define design metrics which can provide a guideline for guaranteeing the energy supply sustainability, improving renewable energy utilization, and prolonging the lifetime of the system;

3) How to effectively design the reliable and economical system with considering practical issues.

In this paper, solar energy-powered BS (SEn-BS) system is studied. Motivated by the aforementioned problems, we firstly provide a theoretical basis for modeling and analyzing energy flow behavior of the system and then define the key design metrics for providing a guideline for system designing. Finally, the sizing optimization problem under constraints of design metrics is formulated as the problem of minimizing the capital expenditure (CAPEX) of the system. To solve the optimization problem, an optimal sizing method, where the size of PV panels and the capacity of the battery bank are jointly optimized, is proposed. Specifically, the main contributions are described as follows: 
1) The energy flow behavior of the SEn-BS system is modeled by using stochastic queue model, jointly considering fluctuation of energy generation, nonlinearity of energy storage, and indeterminacy of traffic load. Based on the constructed model, characteristics of energy flow behavior, i.e., energy-harvesting rate, energy consumption interval, and state probability of energy, are analyzed mathematically, which correspond to different parts of the system.

2) Three key design metrics (i.e., service outage probability, solar energy utilization efficiency, and mean depth of discharge) are defined and analyzed, which can be employed to evaluate and quantify the system reliability, solar energy utilization, and durability. Then, the expressions of them are derived.

Service outage probability (SoP) is defined as the service outage probability of the SEn-BS system caused by insufficient energy supply, which expresses the system reliability;

Solar energy utilization efficiency (SEUE) represents the rate of the amount of energy stored in the battery bank and consumed by the BS to the amount of harvested solar energy;

Mean depth of discharge (MDoD) is denoted as the energy state of the SEn-BS system. Staying in the state of shallow charge or shallow discharge can effectively prolong the lifetime of the system [19].

3) Under constraints of the defined metrics, the sizing optimization problem is formulated to minimize the CAPEX of the SEn-BS system, which is proved to be NP-hard. To solve it in polynomial time, a CAPEX minimization algorithm based on adaptive generic algorithm (AGA) is proposed, and then the optimum sizes of PV panels and the battery bank of the SEn-BS system are achieved.

The rest of this paper is structured as follows. Mathematical models of SEn-BS system components are provided in Section 2. In Section 3, energy flow behavior of the system is modeled as a stochastic queue. Based on the constructed model, energy behavior characteristics are analyzed mathematically, and then the design metrics are defined and illustrated. In Section 4, the sizing optimization problem is formulated, and it is addressed by the proposed CAPEX minimization algorithm based on adaptive genetic algorithm. Results and analyses are presented in Section 5. Finally, Section 6 wraps up the conclusion.

\section{Mathematical modeling of solar energy-powered base station system components}

As illustrated in Figure 1, a typical SEn-BS system mainly comprises the photovoltaic panels, battery bank, and wireless base station. In the system, energy flow generated by PV panels flows into the battery bank and then is extracted to feed the BS energy demand. In order to analyze energy flow behavior of the system, mathematical models of each system component are presented in the following subsections.

\subsection{Photovoltaic output power model}

As the energy harvesting part of the SEn-BS system, PV panels convert solar energy directly into electricity via photovoltaic effect, and the photovoltaic output power $P_{\text {pan }}$ can be given by [20]:

$P_{\mathrm{pan}}=P_{\mathrm{pan}}^{*} \cdot \eta_{\mathrm{pan}} \cdot\left(\frac{G}{G^{*}}\right) \cdot\left[1+a_{T}\left(T_{\mathrm{amb}}+0.02 G-T_{\mathrm{pan}}^{*}\right)\right]$,

where $P_{\text {pan }}^{*}$ is the rated output power of PV panels under standardized test conditions (STC); $\eta_{\text {pan }}$ is the energy conversion efficiency of PV panels; $G^{*}$ is the solar irradiance under STC and equals $1,000 \mathrm{~W} / \mathrm{m}^{2}$; $G$ is the solar irradiance; $a_{T}$ indicates the temperature coefficient and normally equals $-3.7 \times 10^{-3}\left(1 /{ }^{\circ} \mathrm{C}\right)$ for mono and poly crystalline silicon [21]; $T_{\mathrm{amb}}$ is the ambient temperature; $T_{\text {pan }}^{*}$ is the panel temperature under STC and equals $25^{\circ} \mathrm{C}$. Obviously, the photovoltaic output power is mainly determined by the solar irradiance and the ambient temperature.

\subsection{Energy state model of lithium battery bank}

Battery bank in the SEn-BS system is utilized to store energy generated by PV panels to regulate system voltage and to supply energy to the BS. In this paper, the lithium battery bank is considered, as it owns characteristics of high-energy density, long endurance time, and easy maintenance [22].

Depending on the PV energy production and the BS energy requirements, the energy state of the lithium battery bank can be described as two processes: battery bank charging and discharging.

(1) Battery bank charging,

$$
E_{B}(t)=E_{B}(t-\Delta t)+E_{\mathrm{pan}}(t) \cdot \eta_{B}
$$

where $E_{B}(t)$ and $E_{B}(t-\Delta t)$ are the energy state of the battery bank at time $t$ and $t-\Delta t$, respectively, $E_{\mathrm{pan}}(t)$ is the energy generated by PV panels during the period [ $t-\Delta t, t]$, and $\eta_{B}$ represents the charge efficiency of the battery bank. 


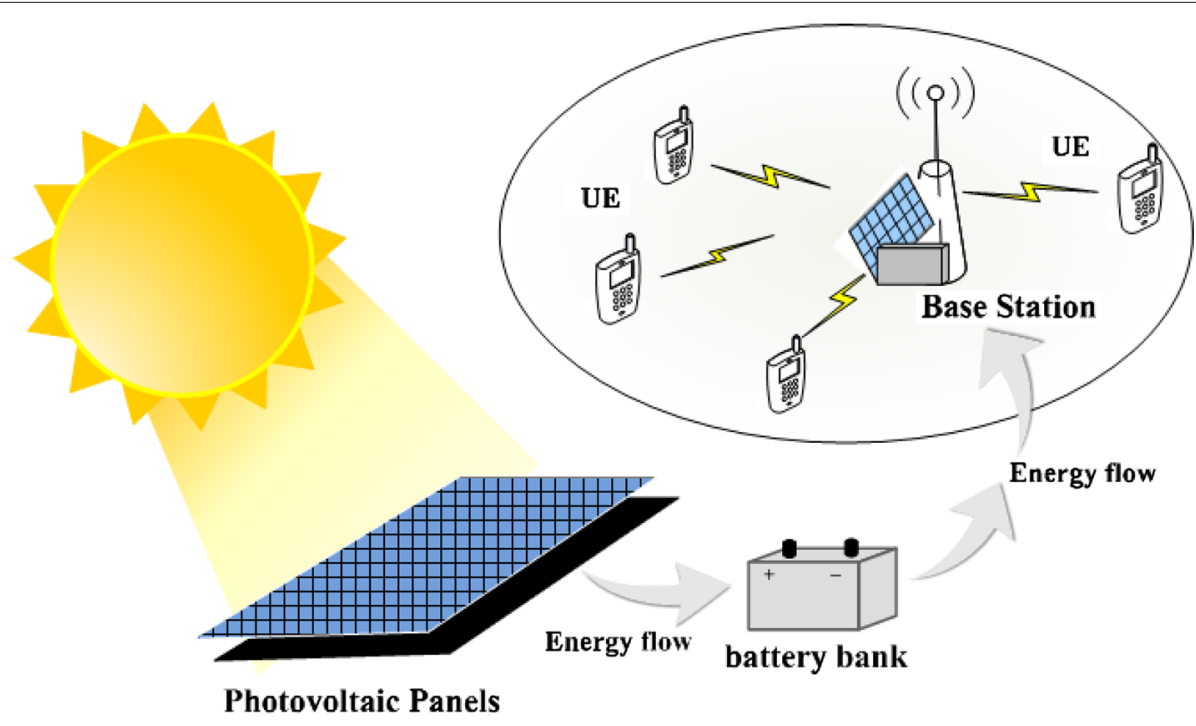

Figure 1 Schematic diagram of the solar energy-powered BS system. A typical SEn-BS system mainly comprises photovoltaic panels, the battery bank, and the wireless base station. In the system, energy flow generated by PV panels flows into the battery bank and then is extracted to feed the BS energy demand.

(2) Considering nonlinear characteristics of the lithium battery [9], the battery bank discharging is separated into two parts: the energy consumption to BS (part 1) and the unavailable energy consumption caused by nonlinear characteristics of the lithium battery bank (part 2). Thus, the battery bank discharging is expressed as [23]:

$$
\begin{aligned}
E_{B}(t)= & E_{B}(t-\Delta t)-\underbrace{E_{\mathrm{BS}}(t) / \eta_{\mathrm{inv}}}_{\text {Part } 1} \\
& -2 \underbrace{2 \int_{t-\Delta t}^{t} \frac{E_{\mathrm{BS}}(\tau)}{\eta_{\mathrm{inv}}} \cdot \sum_{\theta=1}^{\infty} \exp \left[-\beta^{2} \theta^{2}(t-\tau)\right] d \tau}_{\text {Part } 2},
\end{aligned}
$$

where $E_{\mathrm{BS}}(t)$ is the energy consumption of the BS during the period $[t-\Delta t, t] ; \eta_{\text {inv }}$ is the discharge efficiency; $\theta$ is a factor ranging from 1 to $\infty$, and $\beta^{2}$ is a constant related to the diffusion rate within the lithium battery bank, whose value can be determined by data fitting [24].

Moreover, to prevent the battery bank from being overcharged or completely discharged, $E_{B}(t)$ should be restricted by:

$$
E_{B}^{\min } \leq E_{B}(t) \leq E_{B}^{\max }
$$

where $E_{B}^{\max }$ and $E_{B}^{\mathrm{min}}$ are the nominal capacity and the minimum energy quantity of the battery bank, respectively. Refer to [23], $E_{B}^{\mathrm{min}}$ is determined by the maximum depth of discharge (DoD) of the battery bank $D_{\max }$, and is given by $E_{B}^{\min }=\left(1-D_{\max }\right) \cdot E_{B}^{\max }$.

\subsection{Power consumption model of base station}

The power consumption of base station, which can be abstracted into the dynamic part $P_{\text {dyn }}$ and the static part $P_{\text {sta }}$, is expressed as:

$$
P_{\mathrm{BS}}=P_{\mathrm{sta}}+P_{\mathrm{dyn}} .
$$

The static power consumption $P_{\text {sta }}$ exists whenever the SEn-BS system is active, and it can be assumed to be constant [25]. The dynamic power consumption $P_{\text {dyn }}$ is related to the power amplifier and the signal processing unit, and it increases as the number of ongoing connections (users) grows.

Denote the number of users at time $t$ as $\mathcal{M}$. The dynamic power consumption $P_{\text {dyn }}$ can be given by:

$$
P_{\text {dyn }}=\sum_{m=1}^{\mathcal{M}}\left(\frac{P_{m}^{\mathrm{tr}}}{\eta_{\mathrm{PA}}}+P_{m}^{\mathrm{sp}}\right),
$$

where $P_{m}^{\mathrm{sp}}$ is the signal processing power for user $m$, which includes the power dissipation in the mixer, transmit filter, and so on; $P_{m}^{\text {tr }}$ denotes the transmission power of power amplifier over link between user $m$ and its anchored BS, and $\eta_{\mathrm{PA}}$ represents the power amplifier efficiency.

In Equation 6, $P_{m}^{\mathrm{tr}}$ can be further expressed as [26]:

$$
P_{m}^{\operatorname{tr}}=\sigma_{0} \cdot \kappa \cdot d_{m}^{\psi}\left(2^{r_{\min }}-1\right)
$$


where $\sigma_{0}$ is the white Gaussian noise power of the receiver; $\kappa$ represents a parameter to adapt the path loss model; $d_{m}$ denotes the distance between user $m$ and the BS; $\psi \in[2,4]$ is the path loss exponent, and $r_{\min }$ specifies the minimum transmission rate to meet the QoS requirement of users.

Accordingly, substituting Equations 6 and 7 into Equation 5, the power consumption $P_{\mathrm{BS}}$ can be rewritten by:

$$
P_{\mathrm{BS}}=P_{\mathrm{sta}}+\sum_{m=1}^{\mathcal{M}}\left\{\frac{1}{\eta_{\mathrm{PA}}}\left[\sigma_{0} \cdot \kappa \cdot d_{m}^{\psi}\left(2^{r_{\mathrm{min}}}-1\right)\right]+P_{m}^{\mathrm{sp}}\right\}
$$

\section{Modeling and analysis of energy flow behavior and definition of key design metrics in SEn-BS system}

In this section, energy flow behavior of the SEn-BS system is modeled by using a stochastic queue. Then, based on the queue theory, energy characteristics of the energy flow behavior are analyzed mathematically, which set the foundation for the definition and analysis of the key design metrics.

\subsection{Stochastic queue modeling for energy flow behavior}

Influenced by plenty of factors, such as fluctuation of energy harvesting, nonlinearity of energy storage, and indeterminacy of energy consumption, energy flow behavior of the SEn-BS system is regarded as a dynamic and complex process. In this paper, the continuous energy flow behavior is discretized and energy flow is represented by the energy unit, since the mathematical methods associated with discrete coordinate axes make analytical treatment easier than those associated with the continuum.

In the SEn-BS system, the battery bank which is viewed as an energy buffer is utilized to bridge the energy harvesting part (i.e., PV panels) and the energy consumption part (i.e., the base station), c.f. Figure 2. Ng et al. showed that solar energy arrivals can be viewed as a Poisson process [27]. Then, the energy harvesting process can be proven to be a Poisson process due to the deterministic power output model of PV panels. Meanwhile, the energy consumption of the SEn-BS system which depends on plenty of random factors (e.g., traffic characteristics) can also be regarded as a random process. Moreover, owing to the finite capacity of the lithium battery bank, the newly harvested energy units cannot be stored and have to be discarded immediately when the battery bank is full. Therefore, the energy flow behavior of the SEn-BS system is modeled using a $M / G / 1 / K$ queuing system. Note that the capacity of the battery bank is represented to be $K$ energy units.
In order to further dissect the energy flow behavior of the constructed model, representative energy characteristics (i.e., energy harvesting rate, energy consumption interval, and state probability of energy) corresponding to each component of the SEn-BS system are depicted as follows.

\subsection{Energy harvesting rate}

Energy harvesting rate is defined as the mean amount of the harvested energy units per unit time, and energy harvesting process can be viewed as a Poisson process with the energy harvesting rate. In the real SEn-BS system, a maximum power point tracker (MPPT) is usually employed, which makes PV panels keeping the maximum output power in real time [28]. The maximum output power per unit area under STC is denoted as $P_{\text {pan }}^{\max } *$, which can be provided by the manufacturer. Then, the maximum output power of PV panels under arbitrary meteorological conditions, denoted as $P_{\text {pan }}^{\max }$, can be obtained according to Equation 1. Therefore, the effective energy harvesting rate of the SEn-BS system during the period $[0, T]$ is calculated by:

$$
\lambda_{e}=A_{\mathrm{pan}} \cdot \eta_{B} \cdot \frac{1}{T} \int_{0}^{T} P_{\mathrm{pan}}^{\max }(\tau) d \tau
$$

where $A_{\mathrm{pan}}$ is the area of PV panels and $\eta_{B}$ is the battery bank charge efficiency. With a large area of PV panels, more energy may be harvested by the SEn-BS system.

\subsection{Energy consumption interval}

Energy consumption interval represents the period during which an energy unit is consumed. Recalling the lithium battery bank discharging described in Section 2.2, the total energy consumption of SEn-BS system during period $[0, T]$ is given by:

$E_{\mathrm{total}}=E_{\mathrm{BS}} / \eta_{\mathrm{inv}}+2 \int_{0}^{T} \frac{E_{\mathrm{BS}}}{\eta_{\mathrm{inv}}} \cdot \sum_{\theta=1}^{\infty} \exp \left[-\beta^{2} \theta^{2}(T-t)\right] d t$.

where $E_{\mathrm{BS}}$ is the energy consumption of the BS during the period $[0, T]$. Refer to the power consumption model of the BS in Equation 8, $E_{\mathrm{BS}}$ can be calculated by:

$E_{\mathrm{BS}}=P_{\mathrm{sta}} \cdot T+\sum_{m \in \mathcal{U}} t_{m} \cdot\left\{\frac{1}{\eta_{\mathrm{PA}}}\left[\sigma_{0} \cdot \kappa \cdot d_{m}^{\psi}\left(2^{r_{\mathrm{min}}}-1\right)\right]+P_{m}^{\mathrm{sp}}\right\}$,

where $\mathcal{U}$ is the set of active users associated with BS during the period $[0, T]$ and $t_{m}$ is the service time 


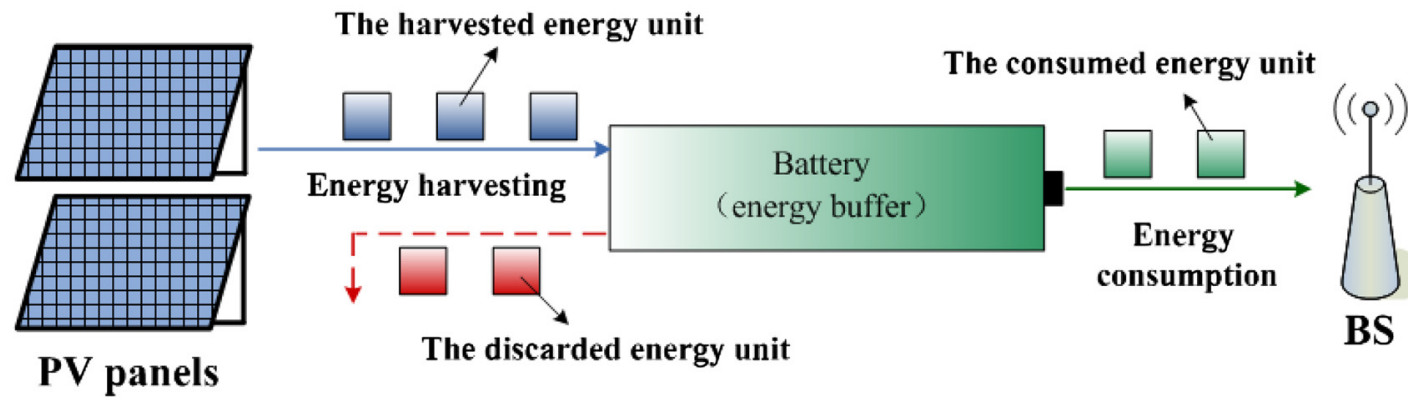

Figure 2 Energy flow behavior modeled by M/G/1/K queue. In the SEn-BS system, energy flow behavior of the SEn-BS system is modeled using a stochastic queue.

of user $m$. Obviously, the total energy consumption of the SEn-BS system is related to plenty of factors, e.g., users' distribution, number of active users, service time of each user, power amplifier efficiency, cell radius, etc.

The cell radius, which indicates the communication coverage of the BS, is one of the most significant parameters in the design stage of the SEn-BS system. It can be adjusted through changing the maximum transmission power level of the BS. In this paper, to facilitate analysis focused on the relationship between the total energy consumption of SEn-BS system and the cell radius, some assumptions are illustrated as follows:

Assumption 1. User equipments (UEs) are randomly dropped within the cell coverage following the uniform distribution. The probability density function (PDF) of $d_{m}$, which is the distance between user $m$ and its anchored BS, can be represented as:

$$
f\left(d_{m}\right)=\frac{2}{R_{\text {cell }}^{2}} d_{m}
$$

where $0 \leq d_{m} \leq R_{\text {cell }} ; R_{\text {cell }}$ is the cell radius.

Assumption 2. In the cellular networks, the traffic (active user) arrivals can be modeled as a Poisson process with the rate $\lambda_{m}$ [29]. Therefore, during period [0,T], the mean number of active users is $\lambda_{m}$. T according to the characteristic of exponential distribution.

Assumption 3. For analytical tractability, the service time of UE $m$ is replaced by the mean value of user service time $\mathbb{E}\left[t_{m}\right]$ and the signal processing power of each user are treated as being equal, i.e., $P_{m}^{s p}=\stackrel{\leftrightarrow}{P}^{s p}, m \in \mathcal{U}$.
On the basis of these assumptions, the total energy consumption of SEn-BS system during $[0, T]$ can be rewritten as:

$$
\begin{aligned}
E_{\text {total }}= & \frac{E_{\text {sta }}+E_{\text {dyn }}}{\eta_{\text {inv }}}+\frac{2 \cdot E_{\text {sta }}}{\eta_{\text {inv }}} \sum_{\theta=1}^{\infty} \frac{1-\exp \left(-\beta^{2} \theta^{2} T\right)}{\beta^{2} \theta^{2}} \\
& +\frac{2 \cdot E_{\text {dyn }}}{\eta_{\text {inv }}} \sum_{\theta=1}^{\infty} \frac{1-\exp \left(-\beta^{2} \theta^{2} \mathbb{E}\left[t_{m}\right]\right)}{\beta^{2} \theta^{2}} .
\end{aligned}
$$

where $E_{\text {sta }}=P_{\text {sta }} \cdot T$ and $E_{\text {dyn }}=\frac{\lambda_{m} \cdot T \cdot \mathbb{E}\left[t_{m}\right]}{\eta_{\mathrm{PA}}}$. $\left[\sigma_{0} \cdot \kappa \cdot d_{m}^{\psi}\left(2^{r_{\text {min }}}-1\right)+\eta_{\text {PA }} \cdot \hat{P}^{\mathrm{sp}}\right]$.

Thus, the energy consumption interval of SEn-BS system during period $[0, T]$, denoted as $\widehat{t_{e}}$, is given by:

$$
\widehat{t_{e}}=\frac{T}{E_{\text {total }}},
$$

which is a strictly monotonic function of $d_{m}$.

Based on the PDF of $d_{m}$ obtained from Equation 12 in Assumption 1, the PDF of $\widehat{t_{e}}$ can be given by:

$$
f\left(\widehat{t_{e}}\right)=\frac{2 T\left(T-\chi_{1} \cdot \widehat{t_{e}}-\chi_{2} \cdot \chi_{4} \cdot{\widehat{t_{e}}}\right)^{\frac{2}{\psi}-1}}{\psi R_{\mathrm{cell}}^{2}\left(\chi_{2} \cdot \chi_{3}\right)^{\frac{2}{\psi}} \cdot{\widehat{t_{e}}}^{\frac{2}{\psi}+1}},
$$

where $\frac{T}{\left(\chi_{1}+\chi_{2} \cdot \chi_{4}+\chi_{2} \cdot \chi_{3} \cdot R_{\text {cell }}^{\varphi}\right)} \leq \widehat{t_{e}} \leq \frac{T}{\chi_{1}+\chi_{2} \cdot \chi_{4}}, \chi_{1}=\frac{E_{\text {sta }}}{\eta_{\text {inv }}}$ $\left(1+2 \sum_{\theta=1}^{\infty} \frac{1-\exp \left(-\beta^{2} \theta^{2} T\right)}{\beta^{2} \theta^{2}}\right), \quad \chi_{2}=\frac{\lambda_{m} T \cdot \mathbb{E}\left[t_{m}\right]}{\eta_{\text {inv }} \cdot \eta_{\mathrm{PA}}}\left(1+2 \sum_{\theta=1}^{\infty}\right.$ $\left.\frac{1-\exp \left(-\beta^{2} \theta^{2} \mathbb{E}\left[t_{m}\right]\right)}{\beta^{2} \theta^{2}}\right), \chi_{3}=\sigma_{0} \cdot \kappa \cdot\left(2^{r_{\min }}-1\right)$ and $\chi_{4}=$

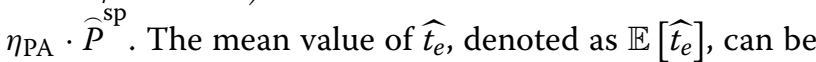
expressed as:

$$
\mathbb{E}\left[\widehat{t_{e}}\right]=\frac{T(\psi+2)}{\left(\chi_{1}+\chi_{2} \cdot \chi_{4}\right) \cdot(\psi+2)+2 \chi_{2} \cdot \chi_{3} R_{\text {cell }}^{\psi}} .
$$


Note that $\mathbb{E}\left[\widehat{t_{e}}\right]$ exponentially decreases while $R_{\text {cell }}$ increases linearly.

\subsection{State probability of energy}

State probability of energy, as a statistical characteristic of energy flow behavior, is defined as the equilibrium probability of energy state in the system at an arbitrary instant of time. Because the battery bank is the unique energy storage device in the SEn-BS system, the energy state of the system can be directly reflected by the amount of energy stored in the battery bank. That is, the energy state of the system can be represented by the number of energy units stored in the battery bank.

Based on the constructed queue model, the state probability of energy is analyzed by using the embedded Markov chain. As shown in Figure 3, the embedded points correspond to energy states (i.e., the number of the stored energy units) when one energy unit is consumed. Assume that there are $E_{B}^{\max }$ energy units in the full lithium battery bank.

Let $\alpha_{k}$ be the probability of $k$ newly harvested energy units during an energy consumption interval. In view of queuing theory [30], with Poisson arrival processes, $\alpha_{k}$ can be obtained as:

$$
\alpha_{k}=\int_{0}^{\infty} \frac{\left(\lambda_{e} \widehat{t_{e}}\right)^{k} e^{-\lambda_{e} \widehat{t_{e}}}}{k !} f\left(\widehat{t_{e}}\right) d \widehat{t_{e}}
$$

where $\lambda_{e}$ and $\widehat{t_{e}}$ can be obtained by Equations 9 and 15, respectively.
Denote the transition probability of energy state from state $i$ to state $j$ as $p_{i j}$. In the embedded Markov chain of Figure 3, the transition probability is expressed as:

$$
\begin{aligned}
& p_{0 j}=\left\{\begin{array}{l}
\alpha_{j} \quad 0 \leq j \leq E_{B}^{\max }-2 \\
\sum_{k=E_{B}^{\max }-1}^{\infty} \alpha_{k} \quad j=E_{B}^{\max }-1
\end{array} \quad i=0\right. \\
& p_{i j}= \begin{cases}\alpha_{j-i+1} \quad i-1 \leq j \leq E_{B}^{\max }-2 \\
\sum_{k=E_{B}^{\max }-i}^{\infty} \alpha_{k} \quad j=E_{B}^{\max }-1\end{cases}
\end{aligned}
$$

Obviously, Equation 18 can be worked out if $\alpha_{k}$ is known.

Let $p_{j}\left(j=0,1, \cdots, E_{B}^{\max }-1\right)$ be the energy state probability immediately after one energy unit consumption. According to the transition probability of energy state in Equation 18, $p_{j}$ can be calculated by solving the balance equations $p_{j}=\sum_{i=0}^{E_{B}^{\max }-1} p_{i j} p_{i}\left(j=0, \cdots, E_{B}^{\max }-1\right)$ and the normalized equation $\sum_{j=0}^{E_{B}^{\max }-1} p_{j}=1$. Since there are $E_{B}^{\max }$ probabilities needed to be worked out, only $E_{B}^{\max }-1$ independent balance equations and the normalized equation are chosen. The set of $E_{B}^{\max }$ linear equations can be summarized as:

$$
\left\{\begin{array}{l}
p_{j}=p_{0} \alpha_{j}+\sum_{i=1}^{j+1} p_{i} \alpha_{j-i+1} \quad j=0,1, \cdots E_{B}^{\max }-2 \\
\sum_{j=0}^{E_{B}^{\max }-1} p_{j}=1
\end{array} .\right.
$$

Let $\mathbf{p}$ be a vector of all probabilities of energy state at the instant of time when one energy unit is consumed, $\mathbf{p}=$

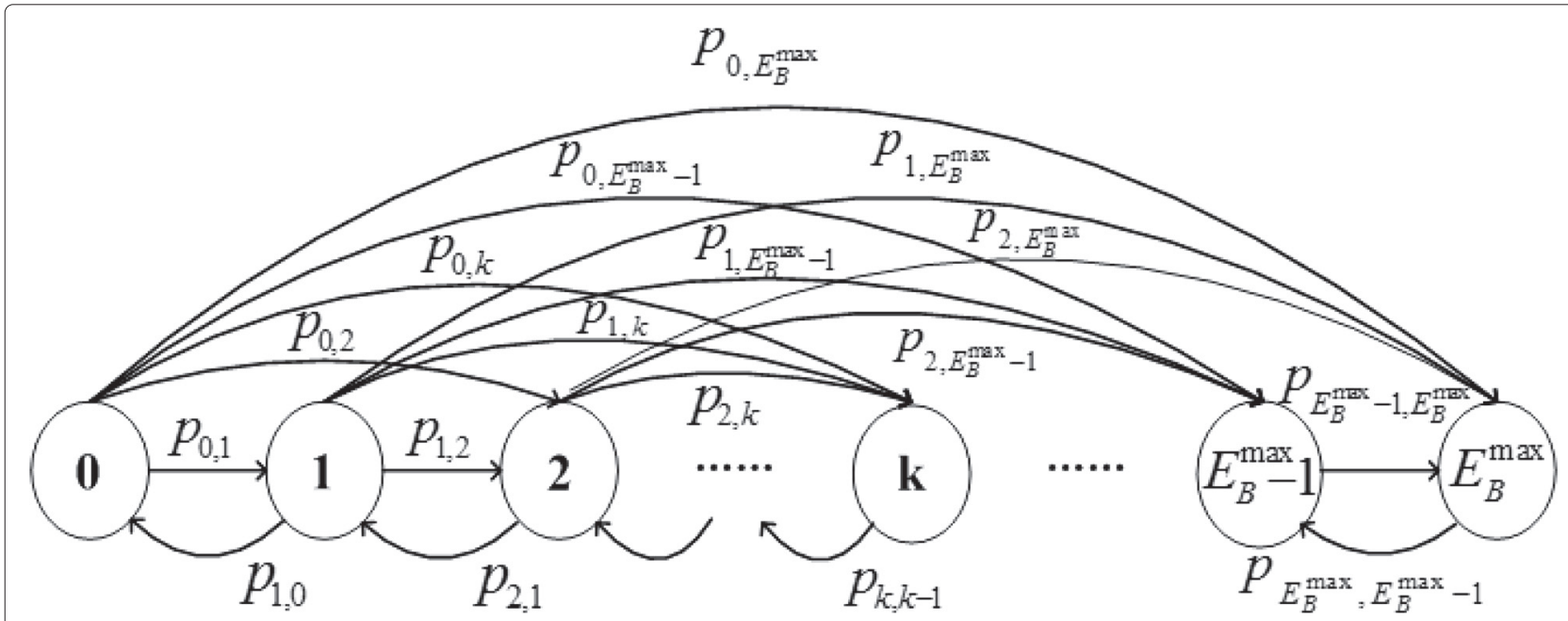

Figure 3 Embedded Markov chain for energy states when one energy unit is consumed. Based on the constructed queue model, the state probability of energy is analyzed by using the embedded Markov chain. 
$\left(p_{0}, p_{1}, \cdots, p_{E_{B}^{\max }-1}\right)^{\mathrm{T}}$, and $\mathbf{b}$ is a vector defined as $\mathbf{b}=$ $(0,0, \cdots 1)^{\mathrm{T}}$. The coefficient matrix $\mathbf{G}$ is expressed as:

$$
\mathbf{G}=\left(\begin{array}{cccccc}
\alpha_{0}-1 & \alpha_{0} & 0 & \cdot & \cdot & 0 \\
\alpha_{1} & \alpha_{1}-1 & \alpha_{0} & \cdot & \cdot & 0 \\
\cdot & \cdot & \cdot & \cdot & \cdot & \cdot \\
\alpha_{E_{B}^{\max }-2} & \alpha_{E_{B}^{\max }-2} & \cdot & \cdot & \alpha_{1}-1 & \alpha_{0} \\
1 & 1 & 1 & 1 & 1 & 1
\end{array}\right)
$$

Equation 19 can be rewritten by $\mathbf{p}=\mathbf{G}^{-1} \mathbf{b}$. Thus, energy state probabilities $p_{j}\left(j=0,1, \cdots, E_{B}^{\max }-1\right)$ are obtained.

The equilibrium probability of energy state in the SEnBS system at an arbitrary instant of time is denoted as $\widehat{p}_{j}$ $\left(j=0,1, \cdots E_{B}^{\max }\right)$. According to the PASTA [30], $\widehat{p}_{j}$ can be derived as:

$$
\widehat{p}_{j}=\left\{\begin{array}{ll}
\frac{p_{j}}{p_{0}+\rho}, & j=0,1, \cdots, E_{B}^{\max }-1 \\
1-\frac{1}{p_{0}+\rho}, & j=E_{B}^{\max }
\end{array},\right.
$$

where $\rho=\lambda_{e} \cdot \mathbb{E}\left[\widehat{t_{e}}\right]$. Obviously, the equilibrium probability of energy state $\widehat{p}_{j}$ is closely related to the energy harvesting rate and the energy consumption interval.

\subsection{Key design metrics definition and analysis}

Based on analysis of energy flow behavior in previous subsections, three key design metrics, i.e., service outage probability, solar energy utilization efficiency, and mean depth of discharge, are proposed. They are a series of numerical measures utilized to assess design quality attributes, such as system reliability, solar energy utilization, and durability.

\subsubsection{Service outage probability}

Guaranteeing the QoS requirement of users is the prior aspect that we ought to consider, because it determines whether the renewable energy can be widely applied in cellular networks. In order to meet the QoS or data rate of users, the energy stored into the battery bank should be above a certain level.

Thus, the SoP is defined as the probability that the energy state of the SEn-BS system is lower than the minimum energy quantity of the battery bank. Denote the minimum energy quantity of the battery bank as $E_{B}^{\mathrm{min}}$. The SoP of the SEn-BS system, denoted as $p_{\text {sop }}$, can be obtained by:

$$
p_{\text {sop }}=\operatorname{Pr}\left(j \leq E_{B}^{\min }\right)=\sum_{j=0}^{E_{B}^{\min }} \widehat{p}_{j} .
$$

The higher the value of the SoP is, the less reliable the SEnBS system becomes.

\subsubsection{Solar energy utilization efficiency}

Energy utilization is another aspect to be considered in the system design. In the SEn-BS system, a capacity mismatch between PV panels and the battery bank (e.g., oversized PV panels or undersized battery bank) could potentially cause solar energy loss. Conversely, the reasonable capacity match between them could improve solar energy utilization and avoid blind capital spending. Thus, the $\mathrm{SEuE}$, denoted as $\gamma_{\text {seue }}$, is defined as the rate of the total amount of energy units stored in the battery bank and consumed by the BS to the total amount of harvested energy units. It can be derived as:

$$
\gamma_{\text {seue }}=\frac{\lambda_{e}\left(1-\widehat{p}_{E_{B}^{\max }}\right)}{\lambda_{e}}=\frac{1}{p_{0}+\rho} .
$$

Obviously, the higher the SEuE is, the more the harvested energy is utilized.

\subsubsection{Mean depth of discharge}

The MDoD is defined as the rate of the mean number of energy units consumed by the BS to the storage capacity of the battery bank, which indicates the steady energy state of the system. Thus, the MDoD is expressed as:

$$
\gamma_{\operatorname{mdod}}=\frac{E_{B}^{\max }-\sum_{j=0}^{E_{B}^{\max }} j \cdot \widehat{p}_{j}}{E_{B}^{\max }} .
$$

Keeping the battery bank in the state of shallow charge or shallow discharge can effectively extend the battery's life and then prolong the lifetime of the system [19].

\section{Optimal sizing for solar energy-powered base station system}

\subsection{Sizing optimization problem formulation}

The size of energy-supply facilities (the area of PV panels and storage capacity of the battery bank) is undoubtedly one of the most concerned issues in the SEn-BS system, since it is closely related to system energy reliability and capital expenditure (CAPEX). It is clear that the larger the size of energy-supply facilities is, the higher of system reliability and CAPEX will be. In order to research out the best trade-off between system economy and reliability, the sizing optimization problem under constraints of the design metrics is formulated as the CAPEX minimization problem.

$$
\begin{aligned}
\min f & \left(A_{\text {pan }}, E_{B}^{\max }\right)=C_{\text {pan }} \cdot A_{\text {pan }}+C_{B} \cdot E_{B}^{\max }, \\
\text { s.t. } & C 1: p_{\text {sop }} \leq p_{\text {sop }}^{\max }, \\
& \text { C2 }: \gamma_{\text {seue }} \geq \gamma_{\text {seue }}^{\min } \\
& \text { C3 }: \gamma_{\operatorname{mdod}}^{\min } \leq \gamma_{\text {mdod }} \leq \gamma_{\text {mdod }}^{\max } \\
& \text { C4 }: E_{B}^{\max } \geq \widehat{E}_{B} .
\end{aligned}
$$

where $A_{\mathrm{pan}}$ and $E_{B}^{\max }$, respectively, indicate the area of PV panels and the storage capacity of the lithium battery bank; $C_{\text {pan }}\left[\$ / m^{2}\right]$ is the cost per unit in installation of PV 
panels, which includes the cost of PV panels, solar panel support and rental cost required by the additional room; $C_{B}[\$ / W h]$ is the cost per unit in installation battery bank, which includes the cost of battery bank and inverter.

Constraint $\mathrm{C} 1$ specifies the maximum tolerable service outage probability $p_{\text {sop }}^{\max }$ which acts as a reliability constraint for the SEn-BS system. C2 is a constraint on the solar energy utilization of the system. The minimum solar energy utilization efficiency is denoted as $\gamma_{\text {seue }}^{\min }$. Constraint C3 is imposed to guarantee that the system stays in the state of shallow charge or shallow discharge. $\gamma_{\text {mdod }}^{\text {min }}$ and $\gamma_{\operatorname{mdod}}^{\max }$ are the lower and upper bound of the optimum running state range, respectively. $\mathrm{C} 4$ ensures the energy supply continuity during the non-availability period of solar energy source. The minimum storage capacity of battery bank is denoted as $\widehat{E}_{B}$. It can be calculated by:

$$
\widehat{E}_{B}=N_{\text {ad }} \cdot E_{\text {total }}^{\text {daily }} / D_{\max }
$$

where $E_{\text {total }}^{\text {daily }}$ is the total daily energy consumption and $N_{\text {ad }}$ is the number of non-availability days of solar energy source.

The formulated problem can be viewed as a buffer allocation problem, which is difficult to solve in polynomial time. Moreover, it is made all the more difficult by the fact that the constraint functions are not obtainable in closed form.

\subsection{CAPEX minimization algorithm based on adaptive genetic algorithm}

The formulated problem can be effectively resolved by metaheuristic methods, especially by the adaptive genetic algorithm (AGA) [31]. As a directed random search technique, AGA adopts natural evolution strategy and finds out the solution by continually evolving a population of candidate solutions [32], and the evolution process mainly includes selection, crossover, and mutation. Therefore, a CAPEX minimization algorithm based on AGA, as an optimal sizing method of the system, is proposed to resolve the sizing optimization problem. The key components of the CAPEX minimization algorithm based on AGA are depicted as follows.

\subsubsection{Initial population}

The initial population is a set of potential solutions which obeys the restricted conditions described in Equation 25. The size of population is denoted as $L$ and then the initial population $\mathbf{E}_{0}$ is represented as $\mathbf{E}_{0}=\left\{\mathbf{S}_{\mathbf{1}}, \mathbf{S}_{\mathbf{2}}, \cdots, \mathbf{S}_{\mathbf{L}}\right\}$. $\mathbf{S}_{\ell}=\left[A_{\ell, \mathrm{pan}}, E_{\ell, B}^{\max }\right](\ell=1,2, \cdots L)$ is the $\ell$ th candidate solution (individual). $A_{\ell \text {,pan }}$ and $E_{\ell, B}^{\max }$ are variables (genes) of the individual $\mathbf{S}_{\ell}$, and the variable value is called genetic information.

\subsubsection{Fitness calculation}

Fitness is employed to evaluate the performance of each individual in the population. A better individual returns a higher fitness. As a performance measurement criterion, the fitness function establishes a mapping of the value of the objective function to fitness, which plays an important role in the evolutionary process. Motivated by simulated annealing thinking [33], the fitness function carrying out the scale transformation is defined by:

$$
F_{\ell}=\frac{\exp \left\{W(g) \cdot\left[1+f\left(A_{\ell, \mathrm{pan}}, E_{\ell, B}^{\max }\right)\right]\right\}^{-1}}{\sum_{\ell=1}^{L} \exp \left\{W(g) \cdot\left[1+f\left(A_{\ell, \mathrm{pan}}, E_{\ell, B}^{\max }\right)\right]\right\}^{-1}}
$$

$$
W(g)=w_{0} \cdot a_{w}^{g} \quad g=1,2, \cdots, g_{\max }
$$

where $F_{\ell}$ is the fitness of the $\ell$ th individual, $\ell=$ $1,2, \cdots L ; f\left(A_{\ell, \text { pan }}, E_{\ell, B}^{\max }\right)$ is the CAPEX function of the $\ell$ th individual; $W(g)$ is the annealing temperature function of the evolutional generation, where $g$ is the current generation number. In Equation 28, $w_{0}$ is the initial annealing temperature and equals $2 \cdot g_{\max } ; g_{\max }$ is the maximum number of generations; $a_{w}$ denotes the annealing temperature coefficient whose value is slightly less than 1.

\subsubsection{Survivor selection}

The fundamental principle of the genetic algorithm is the natural selection [32]. It says that high potential individuals (parents) will produce better ones (offspring). Individuals in the population are selected to undergo crossover and mutation operations for reproduction using the roulette wheel selection [34]. This selection method is based on the distribution obtained by Equation 27. According to the theory of roulette wheel selection, a better individual should have a higher chance to be selected. Consequently, after survivor selection, the better individuals are survived and the worse will be eliminated.

\subsubsection{Crossover operation}

Crossover operation is employed to reproduce new individuals (offspring) by swapping segments of genetic information of two individuals (parents). In AGA, an adaptive crossover strategy is used to improve the search performance of the algorithm. It is realized by assigning a crossover probability to each crossover operation. The 
adaptive crossover probability $p_{c}$ of individuals is defined by:

$$
p_{c}=\left\{\begin{array}{ll}
k_{1}^{\prime}-\left(k_{1}{ }^{\prime}-k_{2}\right) \frac{\left(F^{\prime}-\bar{F}\right)}{F_{\max }-\bar{F}} & F^{\prime} \geq \bar{F} \\
k_{1}{ }^{\prime} & F^{\prime}<\bar{F}
\end{array},\right.
$$

where $F_{\max }$ and $\bar{F}$ are the maximum and the average fitness of the current generation, respectively; $F^{\prime}$ denotes the higher fitness between the two selected individuals; $k_{1}{ }^{\prime}$ and $k_{2}^{\prime}$ are coefficients fixed at the initialization. When $F^{\prime}$ is higher than $\bar{F}$, the lower crossover probability is adopted to reduce the chance of destruction of excellent individual. When $F^{\prime}$ is lower than $\bar{F}$, the higher crossover probability is used to raise the chance of emergence of new and improved individuals. The crossover operation used in the proposed algorithm is arithmetic crossover [35].

\subsubsection{Mutation operation}

Similar with the gene mutation in genetics, mutation operation is used to change some genetic information of an individual. Each individual in the population has a mutation probability. A low mutation probability may hinder the production of the new individual, which is not conducive to the worse individual evolving. However, a high mutation probability reduces the searching performance of the random searching algorithm and may go against retaining the better. Thus, an adaptive mutation probability is employed to ensure the searching ability. The adaptive mutation probability of individual $\ell$ is denoted as $p_{\ell, m}$ and it is defined by:

$$
p_{\ell, m}=\left\{\begin{array}{ll}
k_{3}{ }^{\prime}-\left(k_{3}{ }^{\prime}-k_{4}{ }^{\prime}\right) \frac{\left(F_{l}-\bar{F}\right)}{F_{\max }-\bar{F}} & F_{l} \geq \bar{F} \\
k_{3}{ }^{\prime} & F_{l}<\bar{F}
\end{array},\right.
$$

where $F_{\ell}$ is the fitness of the individual $\ell ; k_{3}{ }^{\prime}$ and $k_{4}{ }^{\prime}$ are predefined parameters. When the fitness of an individual is higher than the average fitness of the current generation, the lower mutation probability is adopted. And when the fitness of the individual is lower than the average fitness, the higher mutation probability is used. In the proposed algorithm, the mutation operation is Gaussian mutation [36]. Each individual in the population should undergo the mutation operation.

After the operation of selection, crossover, and mutation, a new population is generated. It will repeat the same operations until the maximum number of evolutional generations is reached. Finally, the optimum size of the SEn-BS system, i.e., the optimum area of PV panels and storage capacity of battery bank, can be obtained. Simultaneously, the minimum CAPEX of the system is achieved. A pseudo code description of the CAPEX minimization algorithm is shown in Algorithm 1.

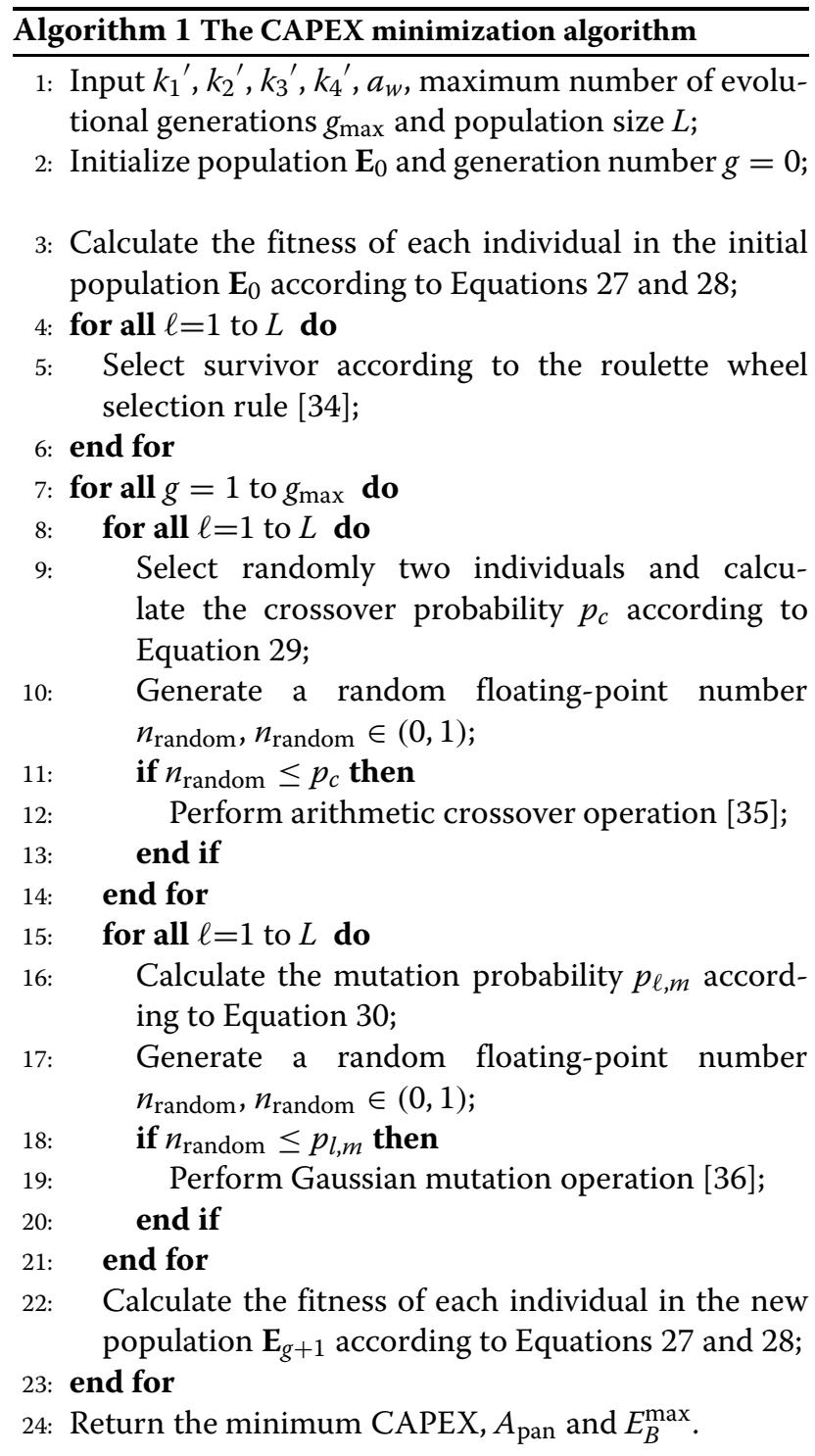

\subsection{Complexity analysis}

Computational complexity is usually used to describe an algorithm's use of computational resources. As for the AGA, we do not consider the computational cost of crossover and mutation because they are constant for each genetic step. The computational complexity is related to the complexity incurred in each iteration and the complexity of evolutional generations. Therefore, the computational complexity is $O\left(L g_{\max }\right)$, where $L$ is the number of population size and $g_{\max }$ is the maximum number of iteration.

\section{Results and analysis}

In this section, we numerically illustrate the design metrics under different system parameters (e.g., area of PV panels, storage capacity of lithium battery bank and cell 
radius of the BS) using the measured meteorological data and traffic data from Shanxi province in China. Furthermore, the proposed optimal sizing method is simulated and is compared with two previously sizing methods described in $[37,38]$. System simulation parameters obtained from $[9,21,39,40]$ are listed in Table 1 .

Figure 4 plots part of the measured meteorological data from Shanxi province, which shows the random distributions of the ambient temperature and solar irradiance. The photovoltaic output power per unit area can be obtained with the measured data according to Equation 1, and then the energy harvesting rate $\lambda_{e}$ over the period $[0, T]$ can be further calculated according to Equation 9. Figure 5 shows the histogram of the statistical data of users' service time and traffic arrival rate, respectively.

These statistical data of cellular networks is collected by one Ericsson's BS (RBS 2206) with the fixed coverage radius $(200 \mathrm{~m})$, which is deployed by China Mobile Communication Corporation in enterprise places. Based on these traffic statistics, the traffic (active user) arrival rate $\lambda_{m}$ and the mean service time of each user $\mathbb{E}\left[t_{m}\right]$ can be

\section{Table 1 System parameters}

\begin{tabular}{|c|c|}
\hline Parameter & Value \\
\hline Carrier frequency/bandwidth & $2.0 \mathrm{GHz} / 10 \mathrm{MHz}$ \\
\hline Path loss model & Table six in [39] \\
\hline Coverage radius of the $\mathrm{BS}$ & $200 \mathrm{~m}$ \\
\hline PA efficiency $\eta_{\mathrm{PA}}$ & $2 / 3$ \\
\hline Minimum downlink transmitted rate for users $r_{\min }$ & $500 \mathrm{Kbps}$ \\
\hline Mean service time of each user $\mathbb{E}\left[t_{m}\right]$ & $108 \mathrm{~s}$ \\
\hline Traffic (active user) arrival rate $\lambda_{m}$ & $0.15\left(m^{2} \cdot h\right)^{-1}$ \\
\hline Signal processing power of each UE $\tilde{P}^{s p}$ & $0.1 \mathrm{~W}$ \\
\hline Static power consumption $P_{\text {sta }}$ & $60 \mathrm{~W}$ \\
\hline Maximum output power per unit area under STC $P_{\text {pan }}^{\max } *$ & $100 \mathrm{~W}$ \\
\hline Energy conversion efficiency of PV panels $\eta_{\text {pan }}$ & 0.95 \\
\hline Charge efficiency $\eta_{B}$ & 0.95 \\
\hline Discharge efficiency $\eta_{\text {inv }}$ & 0.92 \\
\hline Maximum depth of discharge $D_{\max }$ & $80 \%$ \\
\hline Number of non-availability days $N_{\text {ad }}$ & 3 \\
\hline Maximum tolerable service outage probability $p_{\text {sop }}^{\max }$ & 0.01 \\
\hline Minimum solar energy utilization efficiency $\gamma_{\text {seue }}^{\text {min }}$ & $90 \%$ \\
\hline Optimum state range of battery bank $\left[\gamma_{\text {mdod }}^{\min } \gamma_{\text {mdod }}^{\max }\right]$ & $0 \%$ to $10 \%$ \\
\hline Cost per unit in installation of $\mathrm{PV}$ panels $C_{\text {pan }}$ & $35 \$ / m^{2}$ \\
\hline Cost per unit in installation of battery bank $C_{B}$ & $0.4 \$ / W h$ \\
\hline Maximum number of generations $g_{\max }$ & 1,200 \\
\hline Population size $L$ & 200 \\
\hline Crossover operation parameters $k_{1}^{\prime}$ and $k_{2}^{\prime}$ & $0.85,0.6$ \\
\hline Mutation operation parameters $k_{3}^{\prime}$ and $k_{4}^{\prime}$ & $0.1,0.05$ \\
\hline Parameter $a_{w}$ & 0.99 \\
\hline
\end{tabular}

estimated. They are approximately equal to 0.15 times per hour in the unit area and $108 \mathrm{~s}$, respectively.

\subsection{Numerical results of design metrics}

The key design metrics under different system parameters are numerically illustrated as follows.

At first, Figure 6 depicts the SoP, SEuE, and MDoD under different areas of PV panels and cell radii with the given storage capacity of the battery bank (6,000 Ws). Figure 6a shows that the SoP has positive correlation with the cell radius for any given area of PV panels. It is because that the larger the cell radius is, the more the amount of energy is required to guarantee the cell coverage and QoS requirements of cellular users, and it potentially results in the service outage. For any given cell radius, with the area of PV panels increasing, the SoP gradually drops and the system reliability upgrades step by step. In 'Area 1', the SoP remains zero under different areas of PV panels and cell radii. Obviously, it is the optimal design area in term of system reliability.

The SEuE is examined in Figure 6b. Under the same meteorological conditions, PV panels with a large area could harvest more energy, and it is likely to cause the harvested energy to be discarded due to the finite storage capacity of the battery bank. Thus, as the area of PV panels increases, the SEuE reduces for the given cell radius. Nevertheless, for any given area of PV panels, the SEuE increases with the cell radius increasing. 'Area 2' in Figure $6 \mathrm{~b}$ indicates that almost all harvested solar energy is consumed by the BS or stored into the battery bank.

Figure $6 \mathrm{c}$ illustrates the MDoD of the battery bank. It is clear that for the given cell radius, the MDoD decreases with the area of PV panels increasing, and it increases with the cell radius increasing for the given area of PV panels. In 'Area 3', the MDoD of the battery bank is under the $10 \%$ of the storage capacity, and the SEn-BS system stays in the state of shallow charge or shallow discharge, which effectively improves the system running status and prolongs the lifetime of the battery bank and the SEn-BS system.

Then, Figure 7 shows the SoP, SEuE, and MDoD under different areas of PV panels and storage capacities of the lithium battery bank with the given cell radius of the cellular BS (200 m). The SoP is vividly illustrated in Figure 7a. It is obvious that for the given storage capacity of the battery bank, the SoP decreases as the area of PV panels increases. However, different storage capacities have different SoP decreasing rates. For example, when the storage capacity of the battery bank is less than $11,000 \mathrm{~W}$, the SoP declines much smoother than it does when the storage capacity is larger than $11,000 \mathrm{~W}$. This is due to the fact that when the battery bank has a considerable storage capacity, effect of storage capacity on the SoP can be neglected and the SoP varies linearly with the area of PV panels. In 'Area 4', the 


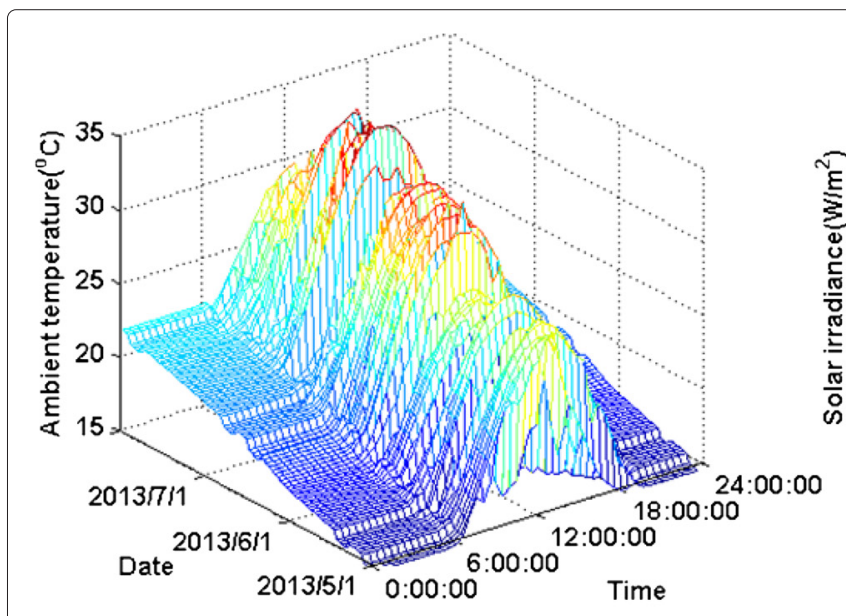

(a)

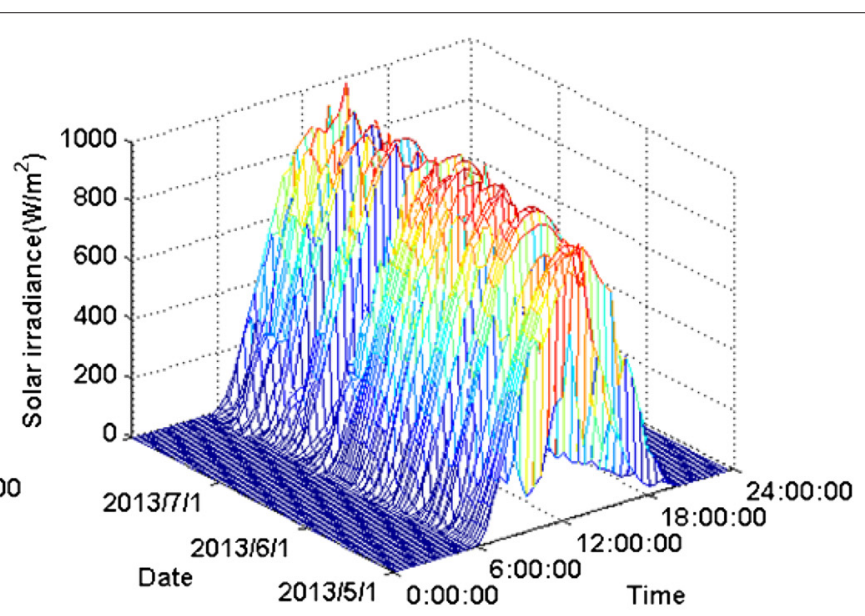

(b)

Figure 4 Measured meteorological data from Shanxi province of China. (a) Measured data of ambient temperature. (b) Measured data of solar irradiance.

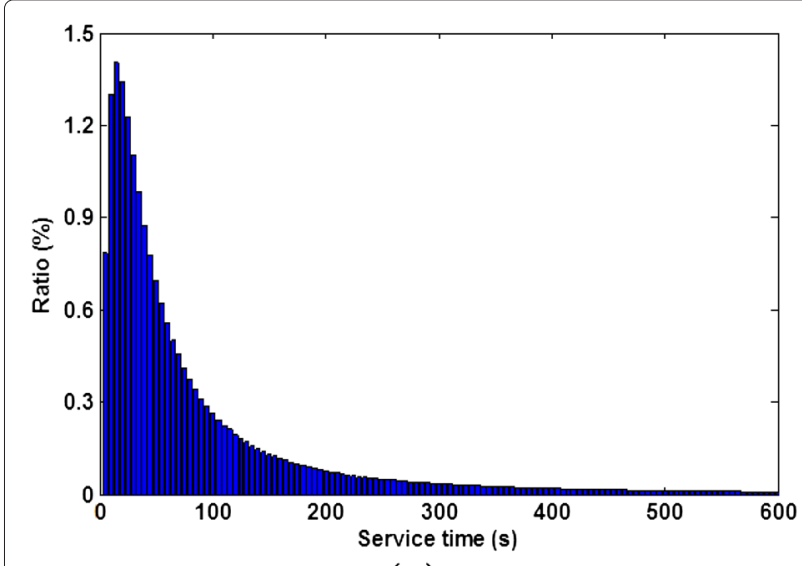

(a)

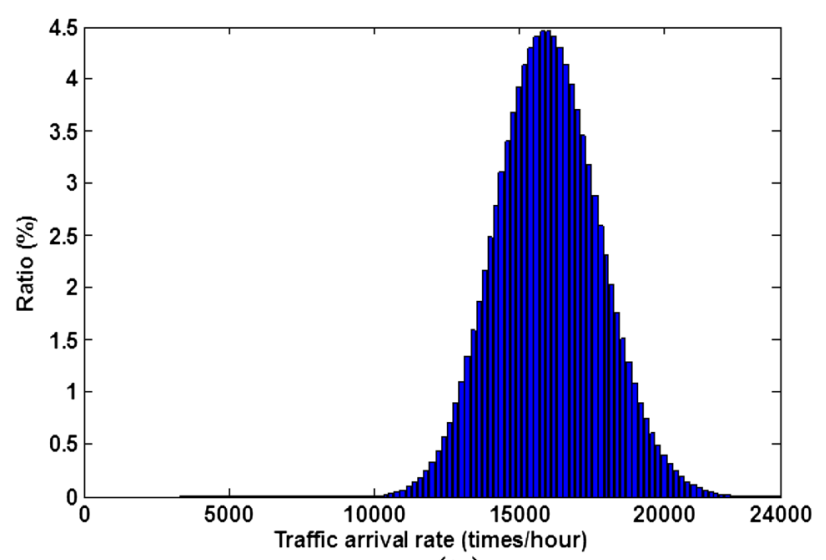

(b)

Figure 5 Statistical traffic data. (a) The histogram of the measured data of service time of active users. (b) The histogram of the measured data of traffic (active user) arrival rate.

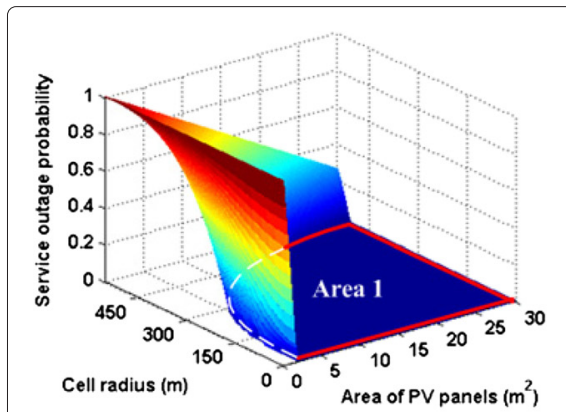

(a)

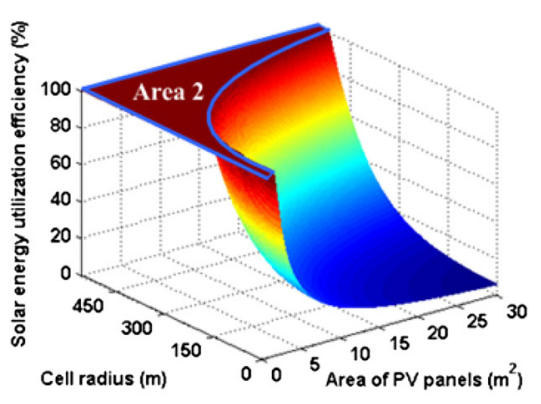

(b)

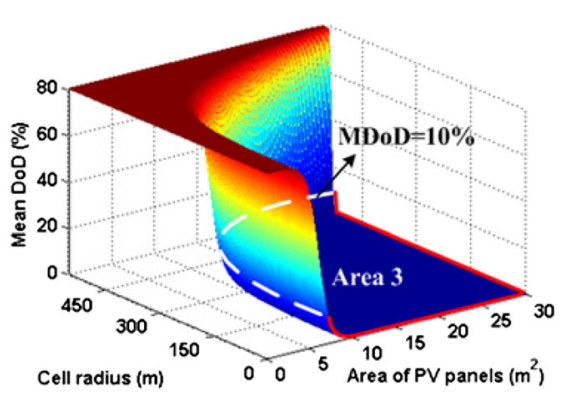

(c)

Figure 6 Design metrics under different PV panel areas and cell radii with the given battery bank. (a) SoP. (b) SEUE. (c) MDOD. 


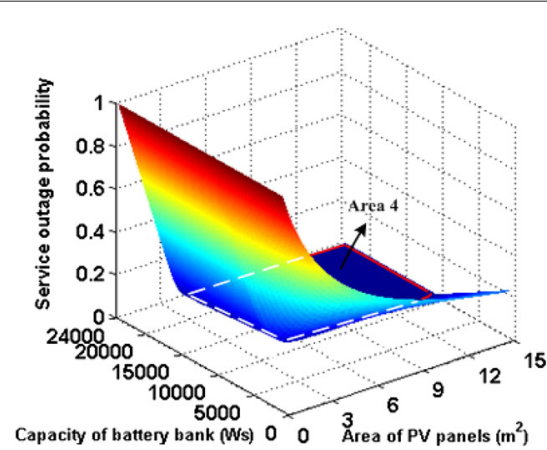

(a)

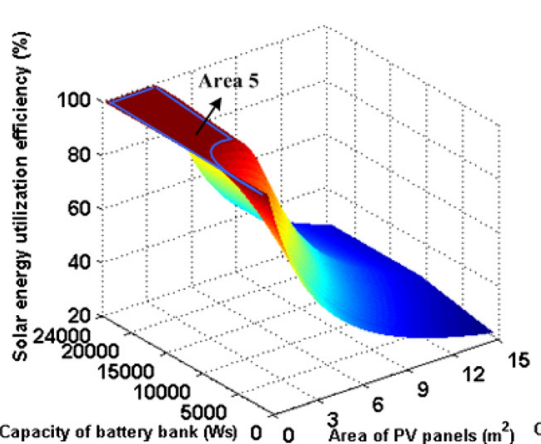

(b)

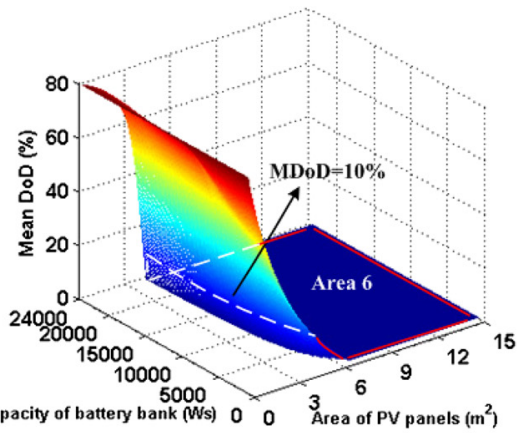

(c)

Figure 7 Design metrics under different PV panel areas and battery banks with the given cell radius. (a) SOP. (b) SEUE. (c) MDoD.

value of SoP remains zero, which means that the energy demand of the BS can be satisfied consistently.

The $\mathrm{SEuE}$ is examined in Figure 7b. With the area of PV panels increasing, the SEuE declines for the given storage capacity. Conversely, with the storage capacity of the battery bank increasing, the $\mathrm{SEuE}$ increases for the given area of PV panels. The reason is that the battery bank with the large storage capacity may provide more room for storing energy and avoid discarding the harvested energy. In 'Area 5', all the harvested energy can be utilized by the SEn-BS system.

Figure 7c shows the MDoD of the battery bank. Based on the MDoD definition, it is clear that as the storage capacity of the battery bank increases, the MDoD slightly grows for the given area of PV panels. However, as the area of PV panels increases, the MDoD declines for the given storage capacity of the battery bank. When the area of PV panels grows to the certain level, the MDoD keeps zero. It indicates that the energy harvesting capacity of the system is far outstripping the BS energy demand. In the 'Area 6', the SEn-BS system works at the optimum state.

At last, Figure 8 illustrates the SoP, SEuE, and MDoD under different storage capacities of the lithium battery bank and cell radii with the given area of PV panels (10 $m^{2}$ ). The SoP and SEuE of the system are vividly shown in Figure $8 \mathrm{a}, \mathrm{b}$, respectively. It is generally known that with a large coverage area, the BS system may serve more users, and thus the more energy may be consumed. Consequently, for the given storage capacity of the battery bank, the SoP and SEuE increase as the cell radius increases. However, with the capacity of the battery bank increasing, the SoP decreases and the SEuE increases for the given cell radius. It is because that the larger the storage capacity of the battery bank, the more the harvested energy can be stored. 'Area 7' and 'Area 8' still indicate the optimal design area in terms of system reliability and energy utilization.

Figure $8 \mathrm{c}$ depicts the MDoD of the battery bank. With the storage capacity increasing, the MDoD decreases for the given cell radius. However, with the cell radius decreasing, the MDoD decreases for the given storage capacity of the battery bank. When the cell radius is less than $400 \mathrm{~m}$, the MDoD remains zero. It is due to the fact that the energy consumption of the BS is much less than the energy supplied by the PV panels and the battery bank. 'Area 8' is the optimal running state area of the SEn-BS system.

Through numerical analysis, the proposed design metrics are closely related to the system parameters, i.e., area

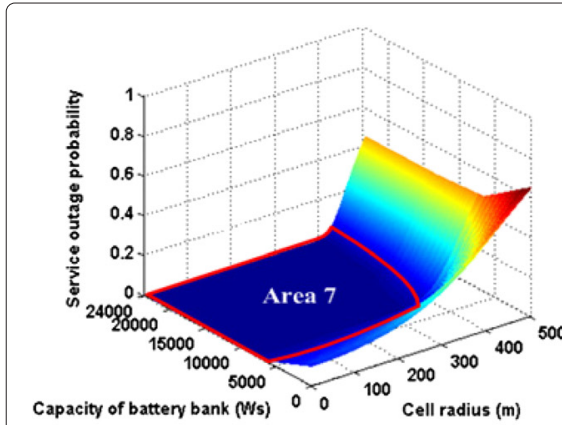

(a)

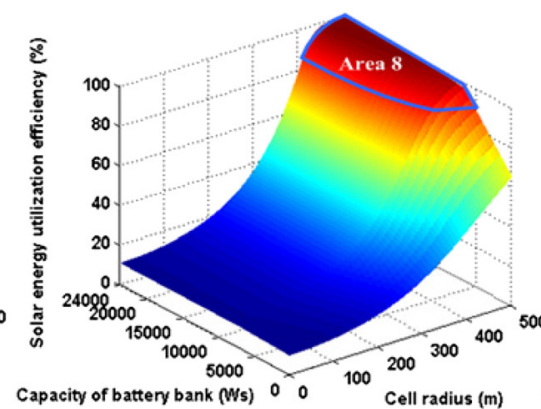

(b)

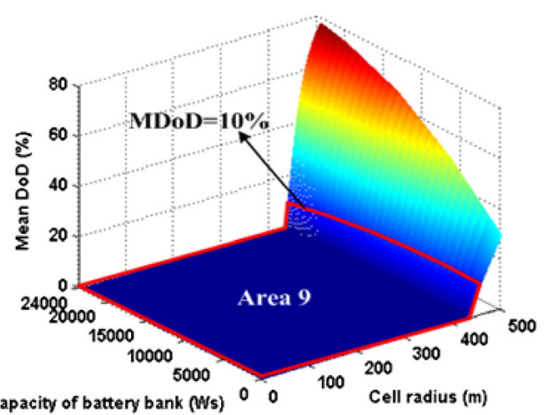

(c)

Figure 8 Design metrics under different battery banks and cell radii with the given PV panel area. (a) SoP. (b) SEuE. (c) MDoD. 


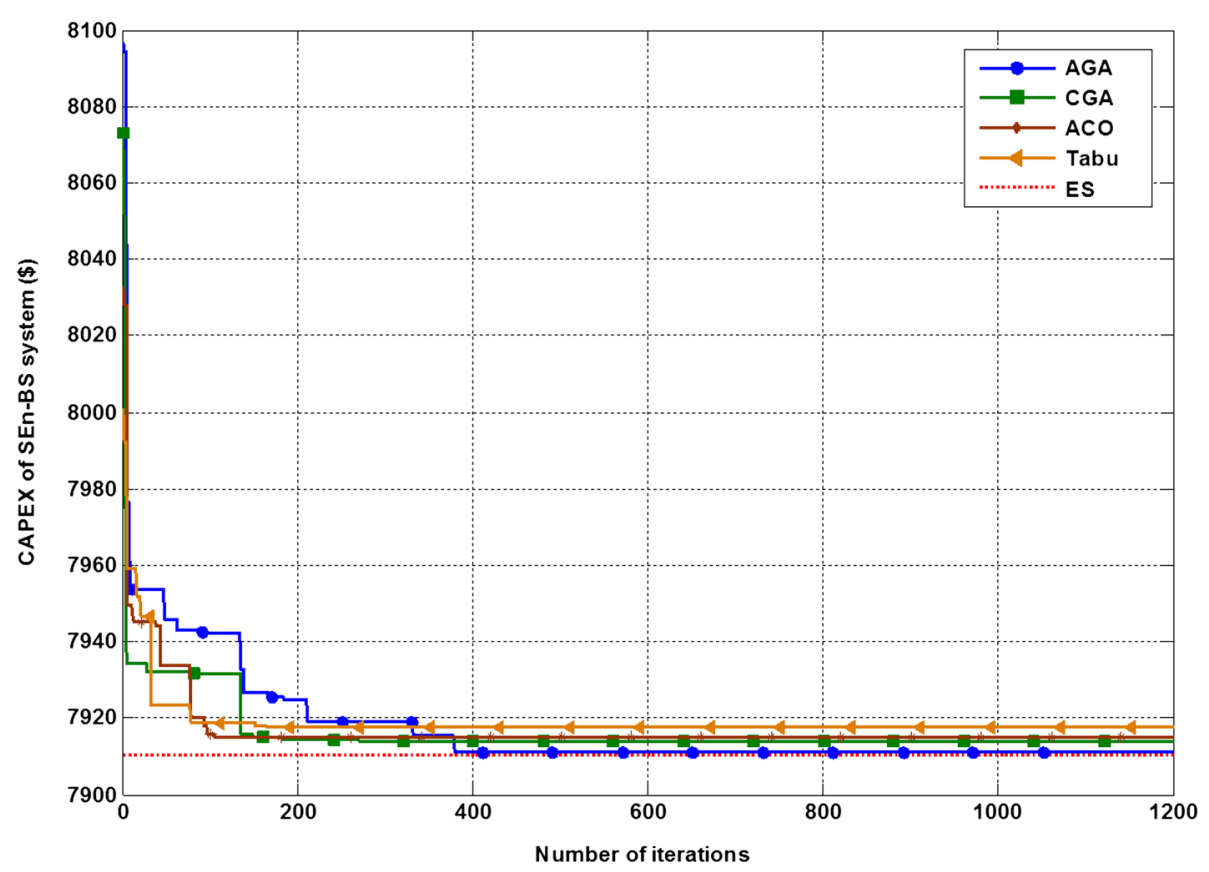

Figure 9 The minimum CAPEX value for different methods.

of PV panels, storage capacity of lithium battery bank and cell radius of the BS. To keep SoP 0\%, SEuE 100\%, and MDoD under $10 \%$, the numerical results provide the optimal design areas in terms of system reliability, solar energy utilization, and durability.

\subsection{Simulation results of CAPEX minimization algorithm} The simulation was carried out on the computer with the Intel Pentium dual CPU (3.20GHz). In Figure 9, the minimum CAPEX of each generation of the AGA, conventional genetic arithmetic (CGA) [41], ant colony optimization (ACO) [42], and tabu search (Tabu) [43] are compared. For the comparison metaheuristic methods, it is very difficult to evolve after a certain number of iterations, whereas the AGA evolves even after 380 generations. This is due to the fact that for the AGA, the fitness function can be adjusted based on the evolutional generations, and the adaptive crossover and mutation operation can improve the search performance. As shown in Figure 9, the minimum CAPEX of the exhaustive search (ES) method, as a baseline, is provided. The difference value of the minimum CAPEX between ES and AGA is $\$ 0.91$, which is smaller than that the difference values between ES and other metaheuristic algorithms. It also indicates that the efficiency of the AGA is higher than that of the comparison metaheuristic algorithms. Furthermore, the running time costs of different algorithms are list in Table 2. It is obvious that the running time between metaheuristic algorithms is not much different, but the ES requires a lot of running time. In this paper, statistical tests have also been conducted to demonstrate the efficiency of the AGA.

In order to compare the performance of the proposed sizing method, two representative sizing methods $[37,38]$ are introduced below.

Comparison method 1: the intuitive sizing method [37]

Step 1: The PV panels' rated power is firstly calculated by the BS's energy consumption, the number of hours in a year, and the number of hours of insolation. Then, the area of PV panels can be further calculated according to the panel's parameters, e.g., energy conversion efficiency.

Step 2: Considering the system reliability, the storage device's nominal capacity is derived by using the storable energy in two extreme sky conditions: several contiguous days of clear skies and several contiguous days of heavily clouded skies.

Step 3: Evaluate the CAPEX related to the PV system by considering the cost of the battery bank and PV plant.

Table 2 Running time of different methods

\begin{tabular}{lc}
\hline Algorithms & Running time (s) \\
\hline AGA & 179.61 \\
CGA & 181.70 \\
ACO & 174.53 \\
Tabu & 150.07 \\
ES & $12,198.43$ \\
\hline
\end{tabular}




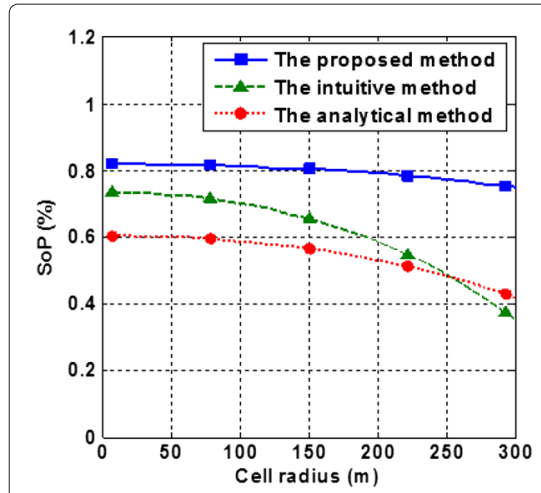

(a)

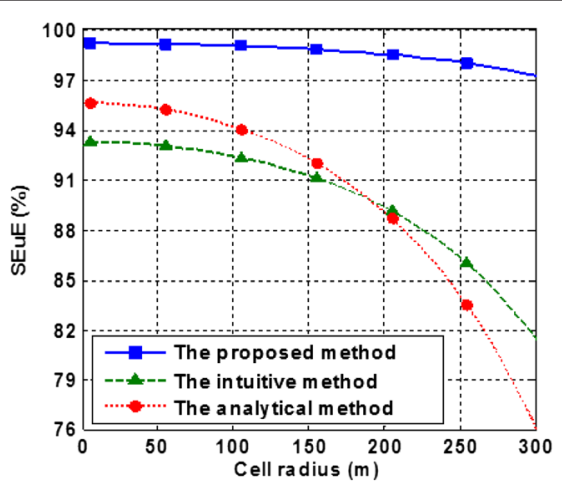

(b)

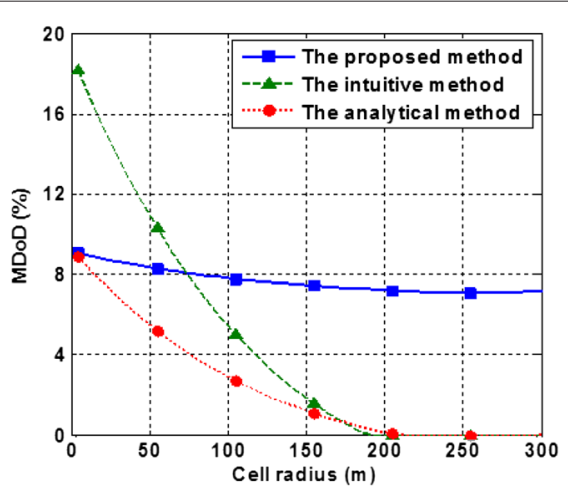

(c)

Figure 10 Comparisons of design metrics of the intuitive method, analytical method, and proposed method. (a) SOP. (b) SEUE. (c) MDOD.

Comparison method 2: the analytical method based on LLP [38]

Step 1: The system reliability is expressed in terms of loss of load probability (LLP) index by means of LLP curves. These curves are expressed by a nonlinear relationship between the area of PV panels and the battery storage capacity. Moreover, the total cost of the PV system installation is also expressed by them.

step 2: The cost of the PV system is closely related to the reliability curve. The optimum area of PV panels and the battery storage capacity are solved by the differential algebraic method.
Figure 10 gives the comparisons of design metrics of the three sizing methods. As shown in Figure 10a, all the SoP values of the three sizing methods are below the maximum tolerable service outage probability 0.01 , but the SoP of the proposed method is slightly higher than other two methods. This is due to the fact that comparison methods only focus on the system reliability and employ the oversized PV panels and battery bank to guarantee the cell coverage and users' QoS requirements. In Figure 10b, the SEn-BS system designed by the proposed method has the higher solar energy utilization. However, the SEuE of other two methods drop sharply with the cell

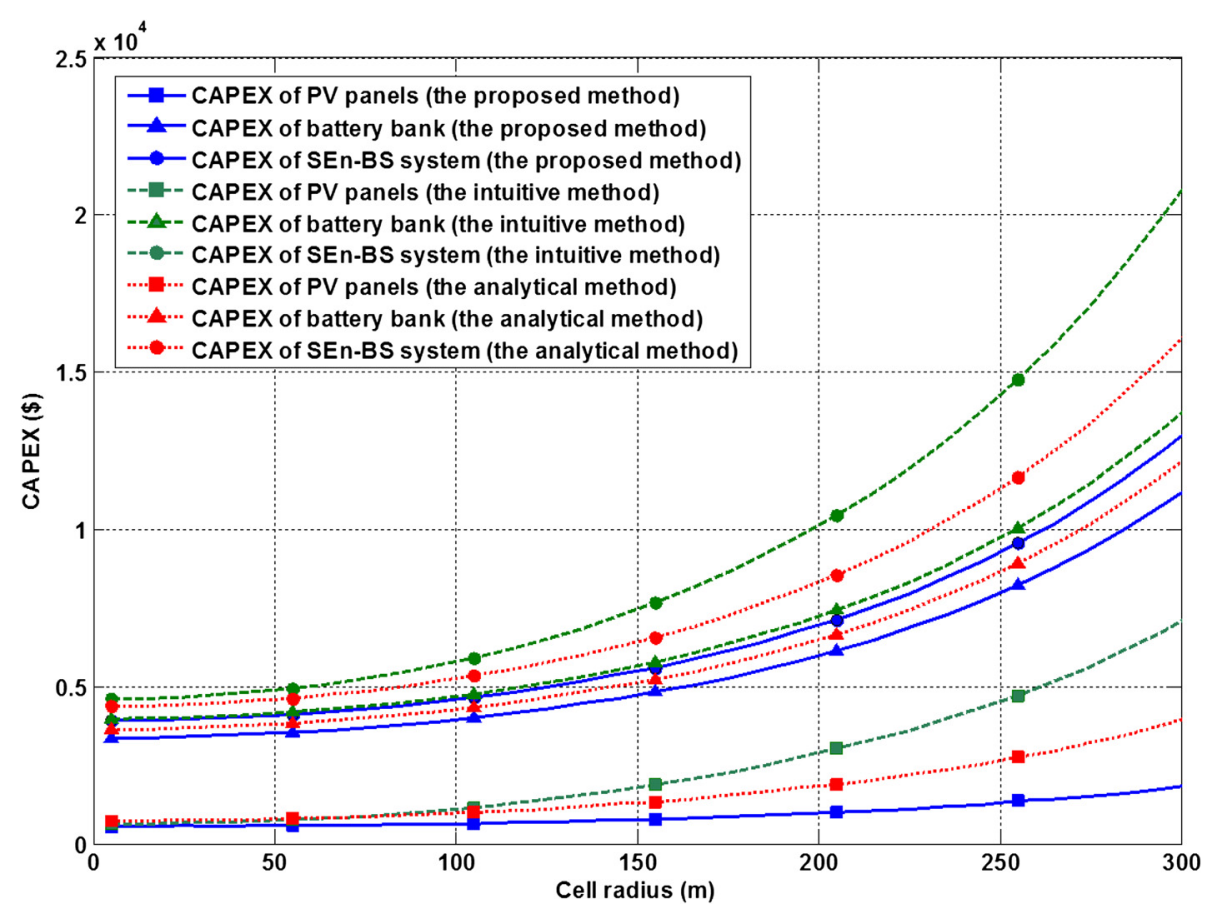

Figure 11 Comparisons of CAPEX of the intuitive method, analytical method, and proposed method. 
radius increasing. For example, for the intuitive method, the analytical method, and the proposed method, when the cell radius is $200 \mathrm{~m}$, the SEuE are $89.5 \%, 89.4 \%$, and $98.3 \%$, respectively. However, when the cell radius is 300 $\mathrm{m}$, the SEuE are $81.8 \%, 76.1 \%$, and $97.2 \%$, respectively. This is due to the fact that in order to feed the BS energy demand, comparison methods rapidly expand the area of PV panels with the increasing of cell radius, and thus it potentially leads to solar energy loss. In Figure 10c, the MDoD of the proposed method and the analytical method remain the optimum running state range, but the MDoD of the intuitive method has the higher value for the small cell radius. The reason is that the intuitive method provides the capacity of PV panels and battery bank without considering the random nature of energy flow.

Figure 11 illustrates three kinds of the CAPEX (i.e., the CAPEX of the SEn-BS system, PV panels, and the battery bank) under the three sizing methods. For the SEn-BS system, in order to guarantee the energy requirement caused by the expanding size of the cellular network, it is necessary to increase the area of PV panels and the storage capacity of the battery bank. Therefore, the CAPEX of both PV panels and battery bank increase as the cell radius grows. For example, for the proposed method, when the cell radius is $150 \mathrm{~m}$, the CAPEX of PV panels and battery bank are $\$ 719.4$ and $\$ 4,453.6$, respectively. When the cell radius is $250 \mathrm{~m}$, they are $\$ 1178.3$ and $\$ 7,277.1$, respectively. Similarly, the same is the case with the comparison methods. However, it is noticeable that these CAPEX of the proposed method are less than those of comparison methods, and the gap increases as the cell radius increases. For the CAPEX of the SEn-BS system, the proposed method saves $15.9 \%, 24.6 \%$, and $33.9 \%$ compared with the intuitive method when the cell radius is 50 , 150 , and $250 \mathrm{~m}$. While it saves $20.3 \%, 22.8 \%$, and $25.9 \%$ compared with the analytical method under cell radius at 50,150 , and $250 \mathrm{~m}$, respectively. This is due to the fact that with the increasing of the cell radius, the corresponding energy harvesting and storage capacity are needed to increase in order to communication reliability. At the same time, dynamic characteristics of energy flow in the SEn-BS system become more obvious, which have significant effects on the accuracy of the sizing design. The proposed sizing method is guided by the efficient design metrics, which obtained by the analyses of energy flow behavior, and thus it achieves significant CAPEX savings. This phenomenon indicates that the proposed method searches out an optimal trade-off between system economy and reliability.

\section{Conclusions}

In this paper, we proposed a theoretical basis for how to design a reliable and efficient SEn-BS system. The energy flow behavior of the system was modeled by using the stochastic queue model, and then dynamics of the constructed model (i.e., energy harvesting rate, energy consumption interval, and state probability of energy) were analyzed mathematically. The definitions and expressions of three key design metrics (i.e., SoP, SEuE, and MDoD) were provided. Under the guidance of these metrics, the optimal sizing method was proposed. Through numerical analysis, the design metrics were closely related to the area of PV panels, storage capacity of the battery bank and cell radius. To keep SOP 0\%, SEuE 100\%, and MDoD under $10 \%$, there exist the optimal design area in terms of system reliability, solar energy utilization, and system durability. Meanwhile, simulation results confirmed that the proposed optimal sizing method, compared with the intuitive and analytical method, has a better performance on guaranteeing system reliability, yielding energy utilization, enhancing system durability, and saving capital expenditures. The proposed modeling, metrics, and optimal sizing method provide a theoretical basis for actual designs of the SEn-BS system, which also can be further applied to the scenario of other renewable energies powered BS system.

\section{Competing interests}

The authors declare that they have no competing interests.

\section{Acknowledgements}

This work was supported by the National Science Foundation of China (No. 61302108), US National Science Foundation (1145596, 0830493).

\section{Author details}

${ }^{1}$ College of Communication Engineering, Chongqing University, 400044 Chongqing, China. ${ }^{2}$ High Performance Network Lab, Institute of Acoustics, Chinese Academy of Sciences, 100190 Beijing, China. ${ }^{3}$ Department of CEEN, University of Nebraska - Lincoln, Lincoln, NE 68182, USA.

Received: 18 May 2014 Accepted: 3 February 2015

Published online: 24 February 2015

\section{References}

1. Hasan Z, Boostanimehr H, Bhargava VK. Green cellular networks: A survey, some research issues and challenges. Commun. Surv. Tutorials IEEE. 2011;13(4):524-540.

2. Wang $L$, Singh C. Multicriteria design of hybrid power generation systems based on a modified particle swarm optimization algorithm. Energy Conversion IEEE Trans. 2009;24(1):163-172.

3. 3GPP. Tech. Specif. Group services and system aspects Telecommunication management; study on energy savings management (ESM) (Release 9), 3GPP TR 32.826. 2009. http://www.3gpp org/ftp/tsg_sa/wg5_tm/TSGS5_68/_SA5_input_to_SA_46/TSsTRs/ 32826/32826-100.doc, Accessed Dec. 2009.

4. 3GPP. Tech. Specif. Group services and system aspects - Evolved universal terrestrial radio access (e-utra); potential solutions for energy saving for e-utran (Release 11), 3GPP TS 36.927. 2012. http://www.3gpp.org/ftp/ Specs/archive/36_series/36.927/36927-b00.zip, Accessed Sep. 2012.

5. Mancuso V, Alouf S. Reducing costs and pollution in cellular networks. Commun. Mag. IEEE. 2011;49(8):63-71.

6. Wang $\mathrm{H}$, Li H, Chen $X$, Qin Y, Yang L, Ci S, Tang H. Liquid cell management for reducing energy consumption expenses in hybrid energy powered cellular networks. In: Wireless Communications and Networking Conference (WCNC), 2014 IEEE. Istanbul, Turkey; 2014. p. 1632-1637. 
7. Chandrasekhar V, Andrews JG, Gatherer A. Femtocell networks: a survey. Commun. Mag. IEEE. 2008;46(9):59-67.

8. Liu S, Lu J, Wu Q, Qiu Q. Harvesting-aware power management for real-time systems with renewable energy. Very Large Scale Integration (VLSI) Syst. IEEE Trans. 2012;20(8):1473-1486.

9. Zhang J, Ci S, Sharif H, Alahmad M. Modeling discharge behavior of multicell battery. Energy Conversion IEEE Trans. 2010;25(4):1133-1141.

10. Cai LX, Liu Y, Luan TH, Shen XS, Mark JW, Poor HV. Sustainability analysis and resource management for wireless mesh networks with renewable energy supplies. Selected Areas Commun. IEEE J. 2014;32(2):345-355.

11. Han T, Ansari N. On optimizing green energy utilization for cellular networks with hybrid energy supplies. Wireless Commun. IEEE Trans. 2013;12(8):3872-3882.

12. Huang $\mathrm{C}$, Zhang $\mathrm{H}$, Cui S. Throughput maximization for the gaussian relay channel with energy harvesting constraints. Selected Areas Commun. 2013;31(8):1469-1479.

13. Sun G, Qiao G, Zhao L. Efficient link scheduling for rechargeable wireless ad hoc and sensor networks. EURASIP J. Wireless Commun. Netw. 2013:1(1):1-14.

14. Sidrach-de-Cardona M, Mora López L. A simple model for sizing stand alone photovoltaic systems. Solar Energy Mater. Solar Cells. 1998;55(3): 199-214.

15. Borowy BS, Salameh ZM. Methodology for optimally sizing the combination of a battery bank and pv array in a wind/pv hybrid system. Energy Conversion IEEE Trans. 1996;11(2):367-375.

16. Yang $H$, Lu L, Zhou W. A novel optimization sizing model for hybrid solar-wind power generation system. Solar energy. 2007;81(1):76-84.

17. Cabral CVT, Diniz ASAC, Martins JH, Toledo OM, Machado Neto LdVB, et al. A stochastic method for stand-alone photovoltaic system sizing. Solar Energy. 2010;84(9):1628-1636.

18. Mellit A. Ann-based ga for generating the sizing curve of stand-alone photovoltaic systems. Adv. Eng. Softw. 2010;41(5):687-693.

19. Jiang $X$, Polastre J, Culler D. Perpetual environmentally powered sensor networks. In: Information Processing in Sensor Networks, 2005. IPSN 2005. Fourth International Symposium On. Los Angeles, California, USA; 2005. p. 463-468.

20. Radziemska $\mathrm{E}$. The effect of temperature on the power drop in crystalline silicon solar cells. Renewable Energy. 2003;28(1):1-12.

21. Ismail M, Moghavvemi M, Mahlia T. Design of an optimized photovoltaic and microturbine hybrid power system for a remote small community: Case study of palestine. Energy Conversion Manag. 2013;75:271-281.

22. Chen S, Gooi HB, Xia N, Wang M. Modelling of lithium-ion battery for online energy management systems. IET Electrical Syst. Transportation. 2012;2(4):202-210.

23. Zhang J, Ci S, Hamid S, Mahmoud A. An enhanced circuit-based model for single-cell battery. In: In Applied Power Electronics Conference and Exposition (APEC). Palm Springs, California, USA; 2010. p. 672-675.

24. Rakhmatov DN, Vrudhula SB. An analytical high-level battery model for use in energy management of portable electronic systems. In: Proceedings of the 2001 IEEE/ACM International Conference on Computer-aided Design. San Jose, California, USA; 2001. p. 488-493.

25. Niyato D, Lu X, Wang P. Adaptive power management for wireless base stations in a smart grid environment. Wireless Commun. IEEE. 2012;19(6): 44-51.

26. Xu Y, Feng Z, Li H, Zhang P, Ci S. Cross-layer design based sustainability and energy-efficiency optimization in femtocell networks with sustainable energy. In: Wireless Communications and Networking Conference (WCNC), 2013 IEEE. Shanghai, China; 2013. p. 152-156.

27. Ng DWK, Lo ES, Schober R. Energy-efficient resource allocation in ofdma systems with hybrid energy harvesting base station. Wireless Communications, IEEE Transactions on. 2013;12(7):3412-3427.

28. Hussein K, Muta I, Hoshino T, Osakada M. Maximum photovoltaic power tracking: an algorithm for rapidly changing atmospheric conditions. IEE Proceedings-Generation Transmission Distribution. 1995;142(1):59-64

29. Wu J, Wu Y, Zhou S, Niu Z. Traffic-aware power adaptation and base station sleep control for energy-delay tradeoffs in green cellular networks. In: Global Communications Conference (GLOBECOM), 2012 IEEE. Anaheim, California, USA; 2012. p. 3171-3176.

30. Cooper RB. Introduction to Queueing Theory. London: Macmillan; 1972.

31. MacGregor Smith J, Cruz F. The buffer allocation problem for general finite buffer queueing networks. IIE Trans. 2005;37(4):343-365.
32. Srinivas $M$, Patnaik LM. Adaptive probabilities of crossover and mutation in genetic algorithms. Syst. Man Cybernet. IEEE Trans. 1994;24(4):656-667.

33. Van Laarhoven PJ, Aarts EH. Simulated Annealing. Netherlands: Springer; 1987.

34. Michalewicz Z. Genetic Algorithms+ Data Structures= Evolution Programs. London: Springer; 1996

35. Michalewicz Z, Janikow CZ, Krawczyk JB. A modified genetic algorithm for optimal control problems. Comput. Math. Appl. 1992;23(12):83-94.

36. Sivanandam S, Deepa S. Genetic Algorithm Optimization Problems. Germany: Springer; 2008.

37. Piro G, Miozzo M, Forte G, Baldo N, Grieco LA, Boggia G, Dini P. Hetnets powered by renewable energy sources: Sustainable next-generation cellular networks. Internet Comput. IEEE. 2013;17(1):32-39.

38. Jakhrani AQ, Othman A-K, Rigit ARH, Samo SR, Kamboh SA. A novel analytical model for optimal sizing of standalone photovoltaic systems. Energy. 2012;46(1):675-682.

39. 3GPP, R4-092042. Simulation assumptions and parameters for FDD HeNB RF requirements, 3GPP TSG RAN WG4 Meeting 51. 2009. http://www. 3gpp.org/ftp/tsg_ran/WG4_Radio/TSGR4_51/Documents/R4-092042.zip, Accessed May 2009.

40. Cao Z, Zhang Z. Parameter settings of genetic algorithm based on multi-factor analysis of variance. In: Genetic and Evolutionary Computing (ICGEC), 2010 Fourth International Conference On. Shenzhen, China; 2010. p. 305-307.

41. Dolgui A, Eremeev A, Kolokolov A, Sigaev $\vee$. A genetic algorithm for the allocation of buffer storage capacities in a production line with unreliable machines. J. Math. Model.Algorithms. 2002;1(2):89-104.

42. Nahas N, Mustapha N, Daoud A-K. Selecting machines and buffers in unreliable series-parallel production lines. Int. J. Production Res. 2009:47(14):3741-3774.

43. Lutz CM, Roscoe Davis K, Minghe S. Determining buffer location and size in production lines using tabu search. Eur. J. Oper. Res. 1998;106(2): 301-316.

\section{Submit your manuscript to a SpringerOpen ${ }^{\circ}$ journal and benefit from:}

- Convenient online submission

Rigorous peer review

- Immediate publication on acceptance

- Open access: articles freely available online

- High visibility within the field

- Retaining the copyright to your article

Submit your next manuscript at springeropen.com 